medRxiv preprint doi: https://doi.org/10.1101/2021.09.11.21263390; this version posted September 13, 2021. The copyright holder for this preprint (which was not certified by peer review) is the author/funder, who has granted medRxiv a license to display the preprint in perpetuity.

All rights reserved. No reuse allowed without permission.

\title{
Rapid realist review: Anxiolytic effects of music therapy on mechanically ventilated patients
}

\author{
Hannah Watson \\ Hull University Teaching Hospital NHS Trust \\ Hull, UK \\ Email: Hannah.watson25@nhs.net \\ (Corresponding author) \\ Patrick Marshall \\ Sheffield Hallam University \\ Sheffield, UK
}




\begin{abstract}

\section{Aims}

To review the literature focusing on anxiety and music therapy as a nonpharmacological anxiolytic for patients receiving mechanical ventilation and to determine the contexts, mechanisms and outcomes of what works for whom and under what circumstances.
\end{abstract}

\title{
Background
}

Mechanical ventilation is one of the numerous processes that a critically ill patient requiring single or multi organ support may experience. This is often frightening and perplexing for the patient, particularly when mechanically ventilated. The abundance of interventions, monitoring and unfamiliar noises can precipitate feelings of stress and anxiety which is common within this population of patients leading to prolonged hospital stay and increases in mortality and morbidity.

\section{Methods}

A rapid realist review (Abrahamson et al. 2020) was undertaken applying the realist methodology to a search of the literature using CINHAL, MEDLINE and Cochrane library, PsyclFNO, PubMed and EMBASE along with searching for the grey literature using an experience library technician, Google Scholar, Google, OpenGrey and the British Library ETHOS.

\section{Results}

Twenty-one studies were included in the review. From the heterogeneity amongst the literature and the poor quality of evidence it was ascertained what worked for whom and under what circumstances. No negative outcomes for patients were noted in the review thus suggesting that music may have a 
place within critical care to help reduce anxiety. Given the heterogeneity of the evidence there is scope to review this topic further.

\section{Conclusion}

The overarching conclusion was that music could help reduce anxiety in the critically ill mechanically ventilated patient. Thematic analysis helped identify what quantifies the markers of anxiety; furthermore, it noted alternative themes that could be explored through more research. Theories could be developed and implemented into a protocol for practice; however, it would be based on the researchers' own experience due to the poor-quality heterogeneous evidence.

Key words: critical care, music therapy, anxiety, non-pharmacological treatments, systematic review, realist methodology 


\section{BACKGROUND}

The abundance of noise, frequent interventions, machinery and monitoring precipitates a frightening and perplexing environment for critically ill patients (Mpouzika et al. 2017). Feelings of psychoemotional distress and anxiety are common amongst this population of patients and this can lead to prolonged hospital stay, mortality and morbidity (Chlan et al. 2013a; Khalaiha et al. 2011; Bradt et al. 2010; Chan et al. 2008; Almured \& Petersson, 2003). Alasad et al. (2015) note that patients in ICU can recall the positives and negative aspects of their stay; however, most recall the most unpleasant aspects, which can lead to posttraumatic stress disorder (PTSD) as noted by Chahraoui et al. (2015) and Wu et al. (2018) noting that a lack of emotional support and the perceived worsening of life and future uncertainty during a patients stay in the Intensive Care Unit (ICU) are a risk factor for posttraumatic stress, anxiety and depression (Barr et al. 2013). There is scope to assess patients' memories, experiences and coping strategies post their stay in ICU; however, it is beyond the scope of this review to evaluate this.

Caine (2003) notes there are links between psychological stress and hypertension, hypotension, pain, cardiac disorders, respiratory pathologies, infection and tachycardia. Papathanassoglou et al. (2010) recognises that psychological stress heightens the pathophysiological sequlae through the release of neuropeptides, which can be measured to assess the stress and effectiveness of psychological support interventions particularly in noncommunicative critically ill patients.

Bradt and Dileo (2014) suggested that music therapy may be beneficial for mechanically ventilated patients and noted a reduction in physiological 
measurements and anxiety in their Cochrane review, which was also noted in other systematic reviews by Umbrello, et al (2019), Davis and Jones (2012), Nilsson, (2008) and Biley (2000).

Sepsis, acute respiratory failure, cardiac failure, pneumonia, trauma and neuromuscular disease are amongst the many pathologies that the critically ill patient often endures requiring single or multiple organ support in particular; mechanical ventilation (Longmore et al. 2014; Loftus, 2010; Waldmann et al. 2008; Cooper et al. 2006). Patients report that the most frustrating part of being mechanically ventilated is the inability to communicate; followed by thirst, difficulty swallowing and pain (Flinterud \& Anderson, 2015; Karlsson et al. 2012; Khalaia et al. 2011). These frustrations can lead to a range of emotions as reported by patients including: panic; fear; anger; helplessness; hopelessness. They report that they feel a loss of control and being overwhelmed in addition to being critically ill (Flinterud \& Anderson, 2015; Karlsson et al. 2012; Khalaia et al. 2011).

This causes pain, anxiety and delirium, particularly in mechanically ventilated patients as they often require pharmacological management to aid with ventilator synchronisation and manage pain, which can be associated with adverse outcomes including prolonged hospital stay, ventilation and further complications increasing morbidity and mortality (Reade \& Finfer, 2014; Barr et al. 2013 Khalaia et al. 2011; Thomas, 2003).

It is common for patients to undergo biological stress, physiological stress and psychological stress; particularly, those undergoing medical interventions (Mpouziki et al. 2017; Salamon et al. 2003). Salamon et al. (2003) notes that when haemostasis within a given organism is adjusted secondary to stimuli, 
this can be classified as biological stress. Allostatic loading (multiple causes of stress) can be pathological if not relieved (Clark et al. 2007; Salamon et al. 2003). Psychological phenomenon of stress is identified through feelings of apprehension, nervousness, anxiety, helplessness (Mpouziki et al. 2017; Salamon et al. 2003). Physiological stress is marked by hypertension, tachycardia, hyperventilation which are linked with ischaemia, fluctuations of body temperature, decreased appetite, enlarged pupils, pain and increased cortisol levels (Mpouzika et al. 2017; Clark et al. 2007; Salamon et al. 2003).

\section{Music and healing}

Music has been recognised for its healing properties for centuries, resulting in positive impacts on human health and well-being throughout history from, ancient Greece through the Christian era, the renaissance, Romanticism and into the $20^{\text {th }}$ century where music therapy was recognised as a professional health care discipline (Davis \& Jones, 2012; Carroll, 2011; Kemper \& Danhauer, 2005, Cardozo, 2004; Darnley-Smith \& Patey, 2003; Biley, 1999). Music has been linked with reducing anxiety, alleviating pain amongst dementia patients, psychiatric patients, patients suffering with anxiety and depression, burns patients, anaesthesiology, patients undergoing biopsy and end of life care (Li et al. 2017; Song et al. 2017; Atiwannapat et al. 2016; Shuman et al. 2016; Gutierrez \& Camarena, 2015; Tanka \& Nogawa, 2015; Brown et al. 2015; Matsota et al. 2013; Crawford et al. 2013; Jiang et al. 2013; To et al. 2013; Wakim et al. 2010; Kemper \& Danhauer, 2005). Davis and Jones (2012) note that in 1978 music therapy was classified as the controlled 
use of music to influence the psychological, physiological and emotional experiences of patients during therapy and rehabilitation.

Music is complex as the mechanisms to how it works is unclear, the thalamus receives musical stimuli of which the effects are transmitted via the reticular activating system (RAS) towards the cerebrum stimulating imagination, intellect, memory and the automatic nervous system (O'Sullivan, 1991). Relaxation, meditation or hypnosis generates alpha waves in the brain indicating the brain is also affected my musical stimulus (O'Sullivan, 1991). Nitric oxide (NO) as a neurotransmitter has a role within the mechanisms of relaxation as it mediates the neuronal pathway from the auditory nerve to the cerebral cortex (Hall, 2016; Lindman \& Chakinala, 2010; Salamon et al. 2003; Galley, 2000). NO as a lipophilic gas is released from the endothelial cells in response to chemical and physical stimuli by relaxing the blood vessels causing vasodilation thus facilitating in lowering blood pressure (Hall, 2016; Lindman \& Chakinala, 2010; Salamon et al. 2003; Galley, 2000). The complexity of human auditory and visual perception, and its link to intelligence are utilised to organise sound in the language self-expression and communication (Carroll, 2011). There are variations of genres of music and its benefits towards the listener (O'Sullivan, 1991). Cultural differences, associations and age offer a wide array of music to suit various people that can trigger positive or negative memories (Kemper \& Danhauer, 2005;

O'Sullivan, 1991). O'Sullivan (1991) notes that the tempo of music has a significant effect on one's psychological state. Steady rhythms with a tempo of $60-70$ beats per minute, low frequency tones and a relaxing melody are found to be soothing and linked with a lower heart rate and respiratory rate (Kemper 
\& Danhauer, 2005; O'Sullivan, 1991). Loud music with a faster rhythm is associated with increased tensions, pain and anxiety; however, it is pertinent to note that music choice should be adapted to the listener's needs and desires (Kemper \& Danhauer, 2005; O’Sullivan, 1991).

\section{Aims and objectives}

The aim of this review was:

- To review the available literature and search for the context, mechanisms and outcomes

- To ascertain the working mechanisms of the intervention

- To examine the theories surrounding the intervention

- To understand how the intervention can be translated into practice and understand the impact for patients

The population, intervention, comparison and outcome (PICO) guide was used to clarify the research questions (Aveyard, 2014):

\section{Population}

The review will focus on mechanically ventilated patients within an intensive care setting; however, for the purpose of theory development studies outside of a critical care setting may be included

Intervention

Music therapy as an adjunct to relieving stress

Comparison

Noise reducing headphones and usual intensive care therapy

\section{Outcome}

The psychological effects, behavioural effects, neurological effects and physiological effects of the patient will be the focus. 


\section{Research questions:}

1. What are the key characteristics of music therapy that help to reduce anxiety? And

2. Does music therapy reduce anxiety in mechanically ventilated patients? And

3. How can this be implemented into practice?

4. What does this mean for patients?

\section{PRISMA}

Meta-analysis and systematic reviews use the Preferred Reporting Items for Systematic Review and Meta-analysis (PRISMA) flow diagram as an evidence-based tool to demonstrate the inclusion and exclusion of articles used. Specifically systematic reviews use the PRISMA for the critical evaluation of a variety of published research (Moher et al, 2009; Liberati et al, 2009). (Refer to diagram 1). Initially this review was undertaken in 2019 therefore an updated search of the literature was carried out on $8^{\text {th }}$ July 2021 to ensure no articles were excluded from this review. Four additional articles were sourced one of which was included for analysis. 


\section{METHODOLOGY}

The clinical experience of working in intensive care settings offer a useful insight for investigating music therapy as an intervention as the overall effect of music is complex. Measuring the physiological effects alone is quantifiable; however, the patient's experiences, feelings and thoughts towards the intervention offer valuable insight into the overall data extraction. Humans are complex and unpredictable and to shed some light on music therapy as an intervention; mixed data and narrative data has offered multiple viewpoints on music as a phenomenon (Polit \& Beck, 2006; Parahoo, 1997). A realist perspective was considered appropriate due to the complexity of the intervention, as the realist seeks to unpick the mechanisms of how the complex programmes/interventions succeed or fail, taking into account the contexts and settings. This review seeks to explore the available evidence rather than to generalise from the research; however, for the purpose of this review the literature has been critiqued to provide examples of the range of evidence available (Wong et al. 2013; Pawson et al. 2006; Pawson et al. 2004). Furthermore it was considered feasible to follow the rapid realist review (RRR) approach due to time constraints and limited resources (Saul et al. 2013).

\section{Rapid realist review (RRR)}

Due to the substantial amount of investment over time a realist review requires which do not always meet the demands of the usual time restricted policy; the RRR is a tool that has been developed to apply a realist approach to a time-sensitive knowledge synthesis process to provide policy makers with a policy that identifies the knowledge and gaps within the literature identifying 
the $\mathrm{CMO}$ configurations in keeping with the core elements of realist philosophy (Wong et al. 2013; Saul et al. 2013; Pawson et al. 2004). This is a streamlined approach that avoids scope creep, which can be an identifiable risk factor for a realist synthesis (Saul et al. 2013). Although the process is like the Cochrane or systematic reviews, RRR is iterative in nature and the initial developments, search strategy, question development and quality approval may be revisited iteratively throughout the RRR process (Saul et al. 2013). RRR is limited as the rapid approach process may miss certain resources and references, which may introduce bias; however, this can be mitigated by using an expert panel or a local reference group ensuring critical elements are not missed (Saul et al. 2013).

\section{Ethics}

Secondary data from established resources is used for this review, therefore did not require ethical approval as it did not involve people taking part in research indirectly and directly.

\section{Data gathering}

This study used a comprehensive literature review; a preliminary search was conducted via search engines using key words to assess the scope and availability of the literature. Further iterative searching assessed the quality and quantity of the literature and identified further key words to be used in the more comprehensive search strategy. 


\section{Search strategy}

CINHAL, MEDLINE, Cochrane library, PsycINFO, PubMed and EMBASE were used to identify published literature along with searching for the grey literature using an experienced library technician, Google Scholar, Google, OpenGrey and the British Library ETHOS.

Boolean logic was applied when identifying key words during the search strategy (Aveyard, 2014): (music therapy* OR music intervention*) AND (critical care OR intensive care OR icu OR ITU) AND (anxiety OR worry OR stress OR fear* OR apprehension OR agitation OR tension)

\section{Inclusion/exclusion}

It is not absolutely necessary in realist synthesis to exclude articles based on their study design, therefore; methodological filters have not been used in this review. Strict inclusion/exclusion criteria are also not a feature of a realist review as searching is iterative because new theories develop in light of emerging data, therefore; inclusion criteria are refined throughout the process of the review (Wong et al, 2013; Pawson et al, 2004). However, an inclusion/exclusion was applied to this review as follows:

Adults (>18yrs), critically ill patients, ventilated patients, intensive care, ITU, ICU, music therapy, English articles, randomised controlled trials (RCT's), cohort studies, Cochrane reviews, pilot studies, interviews and a mixed methods approach were all included in this review $(n=27)$. 
Neonates and children (<18yrs), non-English articles, most articles focusing on non-critically ill patients (if the article focused on anxiety and music therapy in a non-critical ill setting then it was included) and music therapy that focused on relieving pain only were excluded.

Limited time and resources did not enable non-English articles to be included. The focus of this review is adults in critical care and the anxiolytic effect of music therefore children; neonates and non-critically ill patients were excluded to keep this review focused. There is scope to review pain and music therapy as it is linked with anxiety, however the focal point of this review is anxiety due to its subjective nature

Following the search strategy, the research questions were identified using the PICO analysis. Following the scope of the literature common themes were identified which led to further literature searching and further reading which narrowed the number of articles to 21 to be included in this review.

\section{Data extraction}

The realist approach is theory driven to answer multiple questions and through identifying $\mathrm{CMO}$ configurations common themes were identified in the literature (Wong et al. 2013; Saul et al. 2013 Pawson et al. 2006; Pawson et al. 2004; Pawson \& Tilley, 1997).

\section{RESULTS}

The realist approach identifies theories within the mechanisms to delineate the interactions within the context. Refer to Table 1 for a detailed version of CMO configurations and Table 2 for a quick extraction of the data. 


\section{Contexts}

Sixteen of the 21 articles included in this review the main context were adult mechanically ventilated patients within ICU (Almedured \& Petersson, 2003; Beaulieu-Boire et al. 2013; Chan et al. 2008; Chlan, 1998; Chlan et al. 2007; Chlan et al. 2013a; Chlan et al. 2013b; Chlan et al. 2001; Cooke et al. 2010; Han et al. 2010; Korhan et al. 2011; Lee et al. 2017a; Lee, et al. 2017b; Maddpour \& Nematollahi, 2012; Updike, 1990; Wong et al. 2001).

Five of the articles did not focus on mechanical ventilation, Bhana and Botha 2014) and Nilsson (2009) focused on patients undergoing cardiac surgery in ICU and Hamel (2001) focused on patients undergoing cardiac catheterisation. Golino et al. (2019) did focus on adult patients in ICU but had excluded mechanically ventilated patients. Oslon et al (2016) focused on providers use within neurocritical care.

\section{Mechanisms}

The length of the music sessions ranged from 30 to 60 minutes, and all delivered music via headphones using MP3, cassette or CD player barring Golino et al. (2019) who focused their study on the value of active music. Patient directed music was a commonality noted; however, it was from a list chosen by the researchers or a trained music therapist often resulting in a slow tempo and typically relaxing music.

\section{Outcomes}

Anxiety as a phenomenon can be measured in diverse ways as demonstrated throughout the literature and within the context of critically ill mechanically ventilated patients; demi regularities emerged from the data, which identified physiological measurements and state-trait anxiety as the most common 
outcome measurement (Tables $1 \& 2$ ). Thirteen of the 21 studies measured physiological measurements as a marker of anxiety, nine studies noted music had a positive effect on the outcome whereas six of the studies found no effect. Three studies had mixed results; Lee et al. (2017b) found a positive effect on BP HR RR but no effect on DBP, Korhan et al. (2011) noted a positive effect in SBP, DBP and RR but no effect in $\mathrm{HR}$ and $\mathrm{SpO}_{2}$ and Chan et al. (2008) found a positive effect on SBP, DBP, HR and RR in cluster 2 but no effect on DBP, HR, RR in cluster 1 and a positive effect in SBP; furthermore, cluster 1 interestingly was male dominant compared with cluster 2 . This was the only study to note a difference between male and female response suggesting the latter had a more positive effect from music compared with the former.

Fifteen of the studies measured anxiety using validated tools and found that music had a positive effect on the reduction of anxiety; however, three of the 15 studies found no effect in the reduction of anxiety. Biomarkers of stress were measured in five studies and two of the studies found no effect in biomarkers and music therapy. Lee et al. (2017b) found a positive effect in serum cortisol level and Beaulieu-Boire et al. (2013) noted a positive effect in cortisol, prolactin and MET-encephalin levels but no effect in ACTH/cortisol ratio. It is important to note that there is heterogeneity amongst the studies and not one study focused on the same biomarkers of stress.

Patient and health care professional's experiences of music therapy were measured in four studies and all found a positive effect on patients experiences and the role of music in an ICU setting; furthermore, Olson et al. (2016) found a positive effect on practitioners playing music. Music was also 
noted to have a positive effect on sedation in the study by Chlan et al. (2013b). Beaulieu-Boire et al. (2013) and Wong et al. (2001) found no effect on music therapy and analgesia or sedative use.

The overarching results demonstrated that music did not have a negative effect on the outcomes measured suggesting that this is not a harmful mechanism given the context; however, as discussed earlier the quality of evidence is heterogeneous along with the measured outcomes and the mechanisms that lead to the outcomes which does not provide with unambiguous results or a regular pattern.

\section{DISCUSSION}

\section{What works for whom and under what circumstances?}

In keeping with the RRR method, it was difficult to ascertain what works for whom under what circumstances due to the limitations and heterogeneity of the data. The CMO's were teased from the data, which has concluded that the mechanisms and the outcomes are very poorly agreed in the field and more research is needed. It is clear that music can have a place within critical care and there are some suggestions that the most common mechanism is patient directed music for 30 minutes via headphones, which had a positive effect on the outcome. Overall anxiety was reduced, and music is noted to have a positive effect and additionally can do no harm to the patients whether they are receiving mechanical ventilation or not.

\section{Implications and recommendations}

- Music is a cost effective and can be a nurse lead intervention, therefore; a development of a protocol for nurses to follow could be implemented into practice 
- A discussion with the patients' relatives and the patient if they are conscious could be encouraged by the ICU team to establish whether or not music is important to the patient thus arrangements of bringing in the patient's own music with headphones can take place

- It is prudent to note that ICU delirium is prevalent within this population of patients. Music may inhibit the recovery process of those suffering with delirium, therefore; regular CAM-ICU testing should be considered and included into the protocol (Barr et al. 2013)

\section{Limitations}

- One potential non-English article was not included in the review due to a lack of resources available for translation

- One reviewer completing the review, which can lead to errors in the search process, study selection, data analysis and data interpretation.

- Confirmation bias may have occurred but a reflective approach using peers has been used to check for this

- Bias may have also occurred due to the reviewer being an experienced ICU nurse.

- Limited time and resources to complete comprehensive realist synthesis

- Quality of the review may be limited due to the reviewers lack of experience with the RRR methodology

\section{Conclusions}

Adopting the realist approach enabled the horizons to be broadened in a topic which cannot be quantified alone as it requires the response, thoughts, 
feelings and stories from patients to enable a deeper understanding of music as a phenomenon within the critical care setting.

What works for whom and under what circumstances? The answer to this question was contradicted throughout the research as not one paper approached the CMO's the same. Theories could be developed and implemented into a protocol for practice; however, it would be based on the researcher's own experience alongside poor-quality heterogeneous evidence. More research is required with agreed interventions and outcomes with a mixture of qualitative and quantitative evidence, as the complex nature of music as an intervention and humans as a species, our understanding of music as a therapy remains theoretical and very much a personal commodity.

\section{Future research}

Due to the heterogeneity of the evidence, there is scope to conduct further consistent and robust research to enable the development of a protocol that can be applied in practice. Specifically, there is a need for greater definition and agreement around standardized music interventions. Likewise, there is a need for agreed outcomes to measure the effectiveness of these interventions. Finally, as with so many studies in this area, it is essential that attention is paid to the aspects of design including: sample size; and suitable control and comparison groups. 


\section{References}

ABRAHAMSON, V., ZHANG, W., WILSON, P., FARR, W., MALE, I. (2020) Realist Evaluation of Autism ServiCe Delivery (RE-ASCeD): Which diagnostic pathways work best, for whom and in what context? Protocol for a Rapid Realist Review, BMJ Open, 10, 1-8.

ALASAD, J., A., TABAR, N., A. and AHMAD, M., M. (2015). Patient's experience of being in intensive care units. Journal of critical care, 30,859 e7-859e11.

ALMERUD, S. and PETERSSON, K. (2003). Music therapy - a complementary treatment for mechanically ventilated intensive care patients. Intensive and critical care nursing, 19 , 21 30.

ATIWANNAPAT, P., et al. (2016). Active versus receptive group music therapy for major depressive disorder - A pilot study. Complementary therapy in medicine, 26, 141-145.

AVEYARD, H. (2014). Doing a Literature Review in Health and Social Care: A Practical Guide. Third Edition ed., Berkshire, Open University Press.

BARR, J., et al. (2013). Clinical practice guidelines for the management of pain, agitation and delirium in adult patients in the intensive care unit. [online]. Critical care medicine, 41.

BEAULIEU-BOIRE, G., et al. (2013). Music and biological stress dampening in mechanicallyventilated patients at the intensive care unit ward - a prospective interventional randomised crossover trial. Journal of critical care, $28,442-450$.

BHANA, V., M. and Botha, A, D, H. (2014). The therapeutic use of music as experienced by cardiac surgery patients of an intensive care unit. Health SA gezondheid, 19 , 684-693.

BILEY, F., C. (1999). Music as therapy: a brief history. Complimentary therapies in nursing \& midwifery, $5,140-143$.

BILEY, F., C. (2000). The effects on patient well-being of music listening as a nursing intervention: a review of the literature. Journal of clinical nursing, 9 , 668-677.

BRADT, J., DILEO, C. and GROCKE, D. (2010). Music interventions for mechanically ventilated patients. Cochrane database of systematic reviews, (12), N.PAG-N.PAG 1p.

BRADT, Joke and DILEO, Cheryl (2014). Music interventions for mechanically ventilated patients. Cochrane Database Syst.Rev, John Wiley \& Sons, Ltd.

BROWN, B., RUTHERFORD, P. and CRAWFORD, P. (2015). The role of noise in clinical environments with particular reference to mental health care: a narrative review. Internation journal of nursing studies, 52 , 1514-1524.

CAINE, R., M. (2003). Psychological influences in critical care: perspectives from psychoneuroimmunology. Critical care nurse, 23, 60 - 70.

CARDOZO, M. (2004). Harmonic sounds: Complimentary medicine for the critically ill. British journal of nursing, 13, 1321-1324.

CARROLL, D. (2011).

HISTORICAL ROOTS OF MUSIC THERAPY: A BRIEF OVERVIEW

. Revista do núcleo de estudos e pesquisas interdisciplinares em musicoterapia, curitiba, 2 , 171 - 178. 
CHAHRAOUI, K., et al. (2015). Psychological experience of patients 3 months after stay in the intesive care unit: A descriptive and qualitative study. Journal of critical care, $\mathbf{3 0}, 599$ 605.

CHAN, M., F., et al. (2008). Investigating the physiological responses of patients listening to music in the intensive care unit. Journal of clinical nursing, 18, $1250-1257$.

CHLAN, L., L., et al. (2007). Influence of music on the stress response in patients receiving mechanical ventilatory support: a pilot study. American journal of critical care, 16 , $141-145$.

CHLAN, L., L., ENGELAND, W., C. and SAVIK, K. (2013 a). Does music influence stress in mechanically ventilated patients? Intensive critical care nurse, 29, 121 - 127.

CHLAN, L., L., et al. (2013 b). Effects of patient-directed music intervention on anxiety and sedative exposure in critically ill patients receiving mechanical ventilatory support: a randomised clinical trial. Journal of the american medical association, 309 , 2335 - 2344.

CHLAN, L. (1998). Effectiveness of a music therapy intervention on relaxation and anxiety for patients receiving ventilatory assistance. Heart and lung, 27 , 169-176.

CHLAN, L., et al. (2001). Feasibility of a music intervention protocol for patients receiving mechanical ventilatiory support. Alternative therapies, 7 , 80-83.

CLARK, Michael S., BOND, Malcolm J. and HECKER, Jane R. (2007). Environmental stress, psychological stress and allostatic load. Psychology, health \& medicine, 12 (1), 18-30.

COOKE, M., et al. (2010). The effect of music on discomfort experienced by intensive care unit patients during turning: A randomised cross-over study. International journal of nursing practice, $16,125-131$.

COOPER, N., FORREST, K. and CRAMP, P. (2006). Essential guide to acute care . Second Edition ed., Oxford, Blackwell Publishing.

CRAWFORD, I., HOGAN, T. and SILVERMAN, M., J. (2013). Effects of music therapy on perception of stress, relaxation, mood and side effects in patients on a solid organ transplant unit: A randomised effectiveness study. The arts in psychotherapy, 40 , 224-229.

DARNLEY-SMITH, R. and PATEY, H., M. (2003). Music Therapy. London, Sage Publications LtD.

DAVIS, T. and JONES, P. (2012). Music therapy: decreasing anxiety in the ventilated patient: a review of the literature. Dimensions of critical care nursing, 31, 159 - 166.

FLINTERUD, S., I. and ANDERSHED, B. (2018). Transitions on the communication experiences of tracheostomised patients in Intensive Care: A qualitative descriptive study. Journal of clinical nursing, $24,2295-2304$.

GALLEY, HF (2000). Anaesthesia and the nitric oxide-cyclic GMP pathway in the central nervous system. British journal of anaesthesia, 84, 141 - 143.

Golino, A, J., et al. (2019) Impact of an active music therapy intervention on intensive care patients. American journal of critical care, 28, 48-55.

Gutierrez, E, O, F. and Camarena, V, A, T. (2015). Music therapy in generalised anxiety disorder. The arts in psychotherapy, 44 , 19-24. 
HALL, J., E. (2016). Guyton and Hall: Texbook of medical physiology. Thirteenth edition ed., Philadelphia, Elsevier.

HAMEL, W., J. (2001). The effects of music intervetion on anxiety in the patient waiting for cardiac catheterisation. Intensive and critical care nursing, $17,279-285$.

HAN, L., et al. (2010). Effects of music intervention on physiological stress response and anxiety level of mechanically ventilated patients in China: A randomised controlled trial. Journal of clinical nursing, 19 , 978-987.

JIANG, J., et al. (2013). The effects of sedative and stimulatice music on stress reduction depend on music preference. The arts in psychotherapy, 40 , 201-205.

KARLSSON, V., LINDAHL, B. and BERGBOM, I. (2012). Patients' statements and experiences conerning recieving mechnaical ventilation: A prospective video-recorded study. Nursing inquiry, 19 , 247-258.

KEMPER, K., J. and DANHAUER, S., C. (2005). Music as Therapy. Southern american journal, 98 , 282-288.

KHALAILA, R., et al. (2011). Communication difficulites and psychoemotional distress in patients receiving mechanical ventilation. American journal of critical care, 20,470 - 479.

KORHAN, E., A., KHORSHID, L. and UYAR, M. (2011). The effect of music therapy on physiological signs of anxiety in patients receiving mechanical ventilatory support. Journal of clinical nursing, 20 , 1026-1034.

LEE, C., et al. (2017 a). Comparing effects between music intervention and aromatherapy on anxiety of patients undergoing mechanical ventilation in the intensive care unit: a randomised controlled trial. Quality of life research, 26 , 1819-1829.

LEE, C., et al. (2017b). Effects of music intervention on state anxiety and phsiological indices in patients undergoing mechanical ventialtion in the intesive care unit: A randomised controlled trial. Biological research for nursing, 19, 137-144.

LI, J., ZHOU, L. and WANG, Y. (2017). The effects of music intervention on burn patients during treatment procedures: a systematic review and meta-analysis of randomized controlled trials. Complementary and alternative medicine., $17,158$.

LIBERATI, A., et al. (2009). The PRISMA statement for reporting systematic reviews and meta-analysis of studies that evaluate healthcare interventions: explanation and elaboration. (339).

LINDMAN, Brian R. and CHAKINALA, Murali M. (2010). Modulating the nitric oxide-cyclic GMP pathway in the pressure $\_$overloaded left ventricle and group II pulmonary hypertension. International journal of clinical practice, 64 (s168), 15-22.

LOFTUS, I. (2010). Care of the critically ill surgical patient . Third edition ed., Danvers, CRC PressTaylor \& Francis group.

LONGMORE, M., et al. (2014). Oxford handbook of clinical medicine. Ninth edition ed., Oxford, Oxford University Press.

MAHDIPOUR, R. and NEMATOLLAHI, M. (2012). The effect of the music listening and the intensive care unit visit program on the anxiety, stress and depression levels of the heart surgery patient candidates. Critical care nurse, $\mathbf{5}$, 133-138. 
MATSOTA, P., et al. (2013). Music's use for anaesthesia and analgesia. The journal of alternative and complimentary medicine, 19 , 298-307.

MOHER, D., et al. (2009). Preferred Reporting Items for Sytematic Reviews and MetaAnalysis: The PRISMA Statement. 6 , 1-6.

MPOUZIKA, Meropi D. A., GIANNAKOPOULOU, Margarita and PAPATHANASSOGLOU, Elizabeth D. E. (2017). Effects of critical care-related stressors on physiological stress response markers: a pilot study. CONNECT: The world of critical care nursing, 11 (1), 13-17.

NILSSON, U. (2008). The anxiety and pain reducing effects of music interventions: A systematic review. AORN journal, 87 , 781-807.

NILSSON, U. (2009). The effect of music interventionin stress response to cardiac surgery in a randomised clinical trial. Heart and lung, 38 , 201-207.

OLSON, D., M., et al. (2016). Therapeutic use of music and television in neurocritical care: A practice survey. Journal of holistic nursing, $34,6-12$.

O'SULLIVAN, R., J. (1991). A musical road to recovery: music in intensive care. Intensive care nursing., $7,160-163$.

Papathanassoglou, E, D, E., et al. (2010). Potential effects of stress in critical illnes through the role of stress neuropeptides. British association of critical care nurses, $15,204-216$.

PARAHOO, K. (1997). Nursing Research: Principles, Process and Issues. London, Macmillan Press LTD.

PAWSON, R. (2006). Evidence-based policy: A realist perspective. London, SAGE Publications Ltd.

PAWSON, R., GREENHALGH, T. and HARVEY, G. (2006). Realist Review - a new method of systematic review designed for compllex policy interventions. Journal of health services research \& policy, $10,21-34$.

PAWSON, R., et al. (2004). Realist synthesis: an introduction. Manchester, ESRC Research Methods Programme.

PAWSON, R. and TILLEY, N. (1997). Realistic Evaluation. London, SAGE Publications Ltd.

POLIT, D. and BECK, C. (2006). Essentials of nursing research methods, appraisal and utilization . sixth edition ed., Philadelphia, Lippincott Williams \& Wilkins.

READE, M., C. and FINFER, S. (2014). Sedation and delirium in the intensive care unit. New england journal of medicine, $370,444-454$.

SALAMON, E., et al. (2003). Sound therapy induced relaxation: down regulating stress processes and pathologies. Medical science monitor, 9 , 116-121.

SAUL, J., E., et al. (2013). A time-responsive tool for informing policy making: rapid realist review. Implementation science, 8 , 2 - 15.

SHUMAN, J., et al. (2016). Group music therapy impacts mood states of adolecents in a psychiatric hospital setting. The arts in psychotherapy, 49, 50-56. 
SONG, M., et al. (2017). Music for reducing the anxiety and pain of patients undergoing the biopsy: A meta-analysis. Journal of advanced nursing, , 1-14.

TANAKA, Y. and NOGAWA, H. (2015). Evalutating the effects of swinging songs in ethnic music therapy for dementia patients with a novel near-infrared spectroscopy (Data analysis method). International journal of gerontology, 9 , 7-14.

THOMAS, L., A. (2003). Clinical management of stressors perceived by patients on mechanical ventilation. American association of critical care nurses, 14, 73 - 81.

To, W, T, H., et al. (2013). Mozart piano sonatas as a nonpharmacollogical adjunct to facillitate sedation vacation in critically ill patients. Music and medicine, 5 , 119-127.

UMBRELLO, M., et al (2019) Music therapy reduces stress and anxiety in critically ill patients: a systematic review of randomised clinical trials. Minerva Anestesiologica, 85, 886-98

UPDIKE, P. (1990). Music therapy results for ICU patients. Dimensions of critical care nursing, 9 , 39-45.

WAKIM, J., H., SMITH, S. and GUINN, C. (2010). The efficacy of music therapy. Journal of PeriAnesthesia nursing, $25,226-232$.

WALDMANN, C., SONI, N. and RHODES, A. (2008). Oxford desk reference critical care. Oxford, Oxford University Press.

WONG, G., et al. (2013). RAMSES publication standards: realsit synthesis. Journal of advanced nursing, 69,1005 - 1022.

Wong, H, L, C., LOPEZ-NAHAS, V. and MOLASSIOTIS, A. (2001). Effect of music therapy on anxiety in ventilator-dependent patients. Heart and lung, $\mathbf{3 0}, 376-387$.

WU, K.,K., et al. (2018). Posttraumatic stress after treatment in an Intestive Care Unit. East asian arch psychiatry, 28 , 39-44. 


\section{Table 1: CMO Configurations}

\section{Article}

Almerud \& Petersson (2003)

Beaulieu-Boire et al. (2013)

\section{Context}

(Mixed methods approach)

Adult patients on temporary respirator in ICU.

and

Post completion of respirator treatment

investigating the adult patients experience of music therapy.

Randomised crossover study completed in an adult critical care unit. It included patients equiring $>3$ days of mechanical ventilatio and sedation.

\section{Mechanisms}

\section{Outcomes}

The study group $(n=10)$ listened to music via headphones. Classical music was played for 30 minutes in conjunction with a night's sleep.

The control group $(n=10)$ rested under simil circumstances without the headphones or music.

Interviews and questions $(N=6)$ concerning recollections and experiences of respirator treatment and music therapy 2-4 days follow up after patient discharged to the ward.

55 patients were randomised into two group $(A+B)$. Patients in both groups were exposed to the treatment (music - MP3) or the place (sham - MP3) in different random order. Both groups listened to music/placebo for 1 hour twice daily $(a m+p m)$

The music consisted of 10 random

consecutive pieces as advised from a

musicologist.

Blood tests for CRP (C-reactive protein) and
Repeated measures showed no significant differences between the two groups or any differences over time. Paired $t$-tests showed significant mean differences between two

points of measurements on systolic, diastolic blood pressure $(p=0.005)$ and heart rate (increased rate post therapy) $(p<0.002)$ in the study group and not in the control group. No statistical differences were found for respiratory rate and Spo2 for either group.

Recollection:

Patients remembered little of their time on the respirator and none of the patients recalled they had listened to music.

Anxiety and discomfort:

Two patients recalled their experiences of anxiety and discomfort particularly with endotracheal suctioning and the constant noise and lights.

Distinguishing night and day was also a source of distress.

Close relationship:

One patient recounted their sense of security when surrounded by family members and the nursing staff.

Baseline characteristics of both groups were compared using the unpaired $t$ test or the variables.

variables. (arterial blood pressure) heart rate or

respiratory rate over the placebo control.

A trend towards the reduction of narcotics

were noted with music listening ( $p=0.06$ ).

Cortisol and prolactin blood concentration 
Bhana \& Botha (2014)

Chan et al. (2008)
A qualitative research methodology using a contextual, explorative and descriptive research design focusing on cardiac surgery patients in ICU was conducted.

A repeated-measures design was conducted in three ICU's in Hong Kong. major hormonal stress sensors of the HPA axis (prolactin, cortisol, ACTH) were measured every morning pre and post listening period (but not during the evening listening period). Leptin + MET-enkephalin were also measured.

Patient's selected their own music of choice from a selection of music available during the pre-operative period. (Afrikaans,

contemporary, instrumental, Indian classica

During the first 3 days of the post-operative period; patients listened to their choice of

music via a good quality CD/MP3 player with volume control via headphones for periods of 20 minutes at a time during their stay in the ICU.

Once discharged from ICU an unstructured 45 mins interview was conducted by the researcher during the recovery period on the cardiothoracic ward.

Two main outcome variables were measured: demographic measures (age, gender,

religion, education level and previous/current use of relaxation techniques) and

physiological measures (systolic + diastolic

blood pressure, heart rate and respiratory

rate).

Participants listened to music via headphones for 30 minutes. The choice of music was lowpitched with a tempo of 60-80 beats per minute ranging from Chinese classical, religious music and Western classical music. and Western classical).

decreased in music listening group, whereas adreno cortico trophic hormone

$($ ACTH $) /$ cortisol ratio increased $(p=0.02$; $p=0.038+p=0.015$ ). Cortisol responders exhibited reversed associated changes in blood methionine (MET - encephalin) conten $(p=0.01)$

Data analysis consisted of content analysis and coding procedures. Interviews were transcribed verbatim. Data saturation occurred when new data collected was repetitive of that which has already been collected.

Four main themes were identified: practical and operational aspects of music sessions, participants experiences, discomfort due to the therapeutic apparatus and ICU environment and the role of music and

recommendations for music as a therapeutic intervention.

Results identified an overall positive experience and that the sensible and therapeutic use of music is highly beneficial to ICU patients.

Data was coll

A cluster analysis yielded two clusters.

Patients in cluster 1 represented $41.6 \%$ of the

total respondents and typically experienced

low therapeutic effects from listening to

music. Cluster 2 comprised of $58.4 \%$ of the

study sample and reported high therapeutic effects of music.

To conclude the study showed that music

may have more positive effect on groups of

patients whose profile is similar to that of the patients in cluster 2 (more females and older people) and recommend that a clear profile may help health professionals to design appropriate care therapy to target a specific group.

Control group ( $n=27)$ consisted of a 30-minute Independent $t$-tests for mean heart rate, 
Non-sedated adult patients receiving ventilator assistance in four urban Midwestern intensive care units. religious and easy listening were among the

A 2-group experimental design with repeated measures investigated the short-term influence of music on serum levels of biomarkers of the stress response in patients receiving ventilatory support.

A convenience sample of 10 patients receiving mechanical ventilation were recruited from an 11-bedded ICU.

Chlan et al. (2013a)

A randomised controlled trial of patients requiring mechanical ventilation in ICU.

A descriptive pilot study including mechanically ventilated patients within two adult critical care units over three days. rest period.

Experimental group ( $n=27$ ) consisted of selecting a tape from the investigator's collection which consisted of a variety of noncollection a minue classical minute. Classical, new age, country western, choices. Patients listen minutes through headphones via a portsable cassette tape player.

respiratory rate, state anxiety, number of days receiving mechanical ventilation and age no statistic

Independent $t$-tests showed a significant difference between groups on mean posttest state anxiety $(p<0.001)$. There was a greater reduction of posttest state anxiety in the experimental group.

Repeated Measures (RM)- ANOVA showed significant reduction in heart rate over time with the experimental group having a greater reduction $(p<0.001)$.

RM-ANOVA also showed significant reduction of respiratory rate in the experimental group over time $(p<0.001)$.

Patients were randomly assigned to listen to music or to rest quietly for 60 minutes. Levels of corticotropin, cortisol, epinephrine and

No statistically significant differences were detected between groups for heart rate or for any of the serum levels of biomarkers of the 政 4 times during the 60 minutes.

Subjects were randomly assigned to one of three groups:

-experimental patient-directed music intervention (PDM)

-active control condition of noise-cancelling headphones or

-control group of usual ICU care

Five alert mechanically ventilated patients listened to patient-selected music via audiotapes and headphones. The frequency and length of the session was determined by the patient.
The main outcome measure was to measure urinary free cortisol

biomarker of stress.

Mixed model analysis showed no statistical significant differences among groups in UFC over the course of ventilatory support.

Two participants remained in the study whils the other three were weaned and extubated over the study period.

music intervention lasted an average of 67.8 minutes.

Physiological indicators (heart rate,

respiratory rate and blood pressure) and

anxiety have been shown to decrease in

response to music intervention however due 
Chlan et al. (2013b)

Cooke et al. (2010)

Golino et al. (2019)

Hamel (2001)

Han et al. (2010)
A randomised controlled trial including mechanically ventilated patients within ICU from the Minneapolis-St. Paul area.

A single blind randomised cross-over design within ICU across two hospital sites.

A pretest-postest within-subject, single-group design including patients within ICU

A quasi-experimental pretest-postest design investigating the effects of music therapy on anxiety in patient waiting for cardiac catheterisation.

A randomised placebo controlled trial within ICU including 137 mechanically ventilated
Patients were randomised into 3 groups: 1 - patients self-initiated music listening (PDM) with preferred selections tailored by a music therapist.

2 - patients self-initiated use of noise-abating headphones (HP) or

3 - patients received usual ICU care (UC).

Participants listened to music of their choice for 15 minutes prior to turning procedure.

A portable $C D$ player with headphones was used with a choice of jazz, classical, country and we music.

The control arm wore headphones attached to a CP player with no music playing.

Patients were offered a 30-minute music therapy session with a choice of a relaxation/guided imagery intervention ora 'song choice' as guided by a certified music therapist

Subjects listened to music for 20 minutes via a CD player with headphones versus the control group where subjects received treatment as usual.

Intervention group:

Patients received a single 30-minute session to participants not completing the study period there was insufficient data and comparisons were not made.

Mixed model analysis showed a reduction of anxiety in the PDM group compared with the UC group $(p=0.003)$.

PDM reduced sedation intensity and frequency compared to UC + reduced sedation frequency compared to HP groups $(p=0.05+p=0.04)$.

The primary outcome measure for this study was patient discomfort and the secondary outcome measure was anxiety.

There was no significant difference in pre-turn discomfort or anxiety scores between intervention and control groups at period 1 (Wilcoxon's rank sums test, exact $p=0.16$ and $\mathrm{p}=0.31$ ) or between intervention and control groups at period 2 (Wilcoxon's rank sums test, exact $\mathrm{p}=0.85$ and $\mathrm{p}=0.46$ ).

There was no significant difference in pre-turn
The discomfort or anxiety scores between period discomfort or anxiety scores between period and period 2 (Wilcoxon's
exact $p=0.59$ and $p=0.34$ )

Significant decreases were noted in respiratory rate, heart rate, and self reported pain and anxiety levels (for all $=\mathrm{P}<.001$ ).

No significance was noted in oxygen saturations were observed.

The results showed a statistically significan reduction in anxiety $(\mathrm{p}=0.003)$ in the test group alone and in comparing with the contro group $(p=0.004)$. Heart rate and blood pressure was noted to increase in the control group and decrease in the test group.

Comparison of mean difference showed significant differences in heart rate, 
Korhan et al. (2011)

Lee et al. (2017a)

Lee et al. (2017b) unit. patients.

of listening to music of their own choice from the available selection

Placebo group

Patients received headphones and were

minutes. This group did not listen to music.

Control group:

Control group:

Patients were asked to rest with their eyes

closed for 30 minutes without headphones or music.

A study-cased-control, experimental repeated measures design of mechanically ventilated patients within intensive care.

Patients were randomised to a control group or intervention group who received 60 minutes of music therapy via headphones. asked to rest with their eyes closed for 30

respiratory rate, systolic and diastolic blood pressure as well as the Chinese version of Spielberger Strate-Trait Anxiety Scale, but not in saturation of oxygen among the three groups (ranging from $p<0.001$ and $p=0.032$ ) of which greater mean differences were found in the music listening group.

To conclude: The findings confirmed that short term therapeutic effects of music

listening results in substantial reduction in physiological stress responses arising from anxiety in mechanically ventilated patients.

Physiological signs (systolic/diastolic blood pressure, pulse rate, respiratory rate and oxygen saturation) were taken immediately before the intervention and at the $30^{\text {th }}, 60^{\text {th }}$ and $90^{\text {th }}$ minutes of the intervention.

There was a statistically significant difference in mean systolic ( $p=0.024)$ and diastolic ( $p=$ $0.016)$ systolic ( $p$ $0.016)$ blood pressure in both groups. There was a statistically significant difference in the respiratory rate in both groups $(p=0.043)$. There was no statistical difference in the heart rate and in oxygen saturation in both groups.

A randomised controlled trial of mechanically ventilated patients within the intensive care

Patients were randomised to a control group, music intervention group or aromatherapy group.

The music group received 30 minute session each day of their choice via headphones. version of the State-Trait Anxiety Inventory (C-STAl) and the Visual Analogue Scale for Anxiety (VAS-A) at baseline, post-test and 30min follow-up.

Heart rate, respiratory rate and blood pressure were measured every $10 \mathrm{~min}$ from baseline to the 30-min follow-up.

The music group reported a significantly lower level of self-reported anxiety then contro group $(p<0.001)$. The music group had significantly lower heart rate then the control group $(\mathrm{p}<0.001)$.

Anxiety was measured using serum cortisol levels, the C-STAl, the VAS-A, heart rate and blood pressure. After adjusting for demographics, analysis of covariance showed that the music group had significantly better entilated patients within the intensive care unit.
Patients were randomised to intervention or control group. The intervention group liste to music for 30 minutes and the control group wore headphones without music for 30 minutes. 
Mahdipour \& Nematollahi (2012)

Nilsson (2009)

Olson et al. (2016)

Updike (1990)

Wong et al. (2001)
A quasi-experimental pretest-posttest design within a cardiac ICU.

A repeated-measures randomised controlled trial of patients undergoing cardiac surgery.

17-item practice survey distributed electronically to neurocritical care society members.

A pre-post-test design within ICU.

A crossover repeated measures design with random assignment within an ICU setting.
Patients were randomly assigned to either a control group or experimental group, the experimental group received music listening or a visit from the ICU program.

Patients were randomised to the control group or the music group. Patients in the music group listen group. Patients in the pillow connected to an MP3 player.

\section{TV when caring for patient} neurologic injury whilst in ICU.

The patients chose one of eight programs of classical or contemporary music and listened to 30 minutes via headphones.

scores for all posttest measures $(p<0.02)$ and pre-post differences $(P<0.03)$ except for diastolic blood pressure.

Variables were measured pre and post interventions using the Depression, Anxiety and Stress Scale (DASS 21).

Pre intervention showed no statistical difference of the scores of mean DASS 21 ( $p$ $>0.05$ ).

Post intervention there was no statistical difference within the treatment groups but there was a significant variation between treatment and control groups $(p<0.001)$.

After 30 minutes of bed rest there was significant difference in serum cortisol levels between the groups $(p<0.02)$. However; afte 60 minutes there was no diference in serum difference in heart rate, respiratory rate, mean arterial pressure, arterial oxygen tension, arterial oxygen saturation and pain and arterial oxygen saturation and pain
anxiety levels between the groups.

118 completed responses, the majority of respondents sometimes or always play music and agree that it is therapeutic $(70 \%)$. However, there was no clear practice pattern regarding when or why music or TV should be used as an intervention.

A nondirective open-ended questionnaire was used to yield data on patients' emotional

responses before and after the music therapy. A paired t-test analysis showed significant reduction in systolic/diastolic blood pressure, mean arterial pressure.

The results of emotional status indicated the patients' mood shifted significantly towards a more desirable state of well-being.

Patients were randomised to receive either 30 minutes of uninterrupted rest and then 30 
minutes of music therapy or the music therapy first and then the rest period. periods $(P<0.01)$

Blood pressure and respiratory rate showed

no significant differences in the 2 conditions,

however; significant differences were

observed at the end of intervention between the 2 conditions.

No difference was found in participants who had taken sedative or analgesic medications compared with those who had not in relation to mean anxiety score, mean BP values or

$$
\text { mean RR. }
$$


Table $2 \mathrm{CMO}$ configurations at a glance

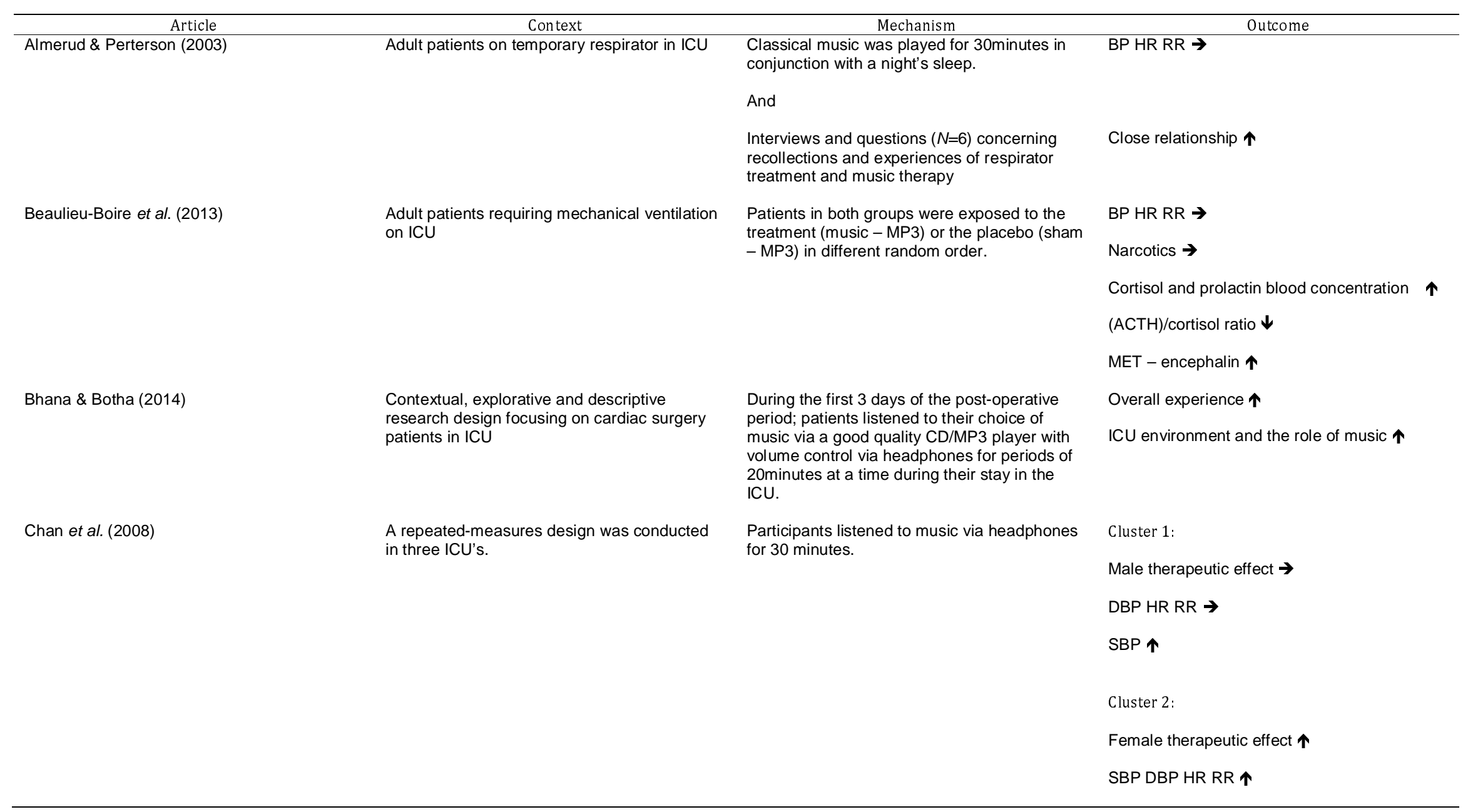


Chlan (1998)

Chlan et al. (2007)

Chlan et al. (2013a)

Chlan et al. (2001)

Chlan et al. (2013b)

Cooke et al. (2010)

Golino et al. (2019)

Han et al. (2010)
Non-sedated adult patients receiving ventilator assistance in ICU

Adult patients receiving mechanical ventilation in ICU

Adult patients requiring mechanical ventilation in ICU.

Mechanically ventilated patients within two adult critical care units

Mechanically ventilated patients within ICU

Adult patients in ICU

Adult patients in ICU

Effect of music therapy on anxiety in patient waiting for cardiac catheterisation.

Mechanically ventilated patients in ICU
Patients listened to the music for 30-minutes through headphones via a portable cassette tape player.

Patients were randomly assigned to listen to music or to rest quietly for 60 minutes

Patient-directed music (PDM)

Alert mechanically ventilated patients listened to patient-selected music via audiotapes and headphones.

Patients self-initiated music listening (PDM)

Participants listened to music of their choice for 15 minutes prior to turning procedure.

Patients received 30 -minute music therapy session of either relaxation intervention or a song choice' intervention

Patients listened to music for 20 minutes via a CD player with headphones

Patients received a single 30-minute session
HR RR over time $\uparrow$

\section{Anxiety $\uparrow$}

Serum levels of biomarkers of the stress response $\rightarrow$

Urinary free cortisol (UFC) $\rightarrow$

BP HR RR $\rightarrow$

Anxiety $\uparrow$

Sedation $\uparrow$

Anxiety $\rightarrow$

$\mathrm{HR} R \mathrm{R} \uparrow$

Anxiety and pain

Oxygen saturations $\rightarrow$

Anxiety $\uparrow$

HR BP $\uparrow$

BP HR RR ^ 


\section{Mahdipour \& Nematollahi (2012)}

Nilsson (2009)

Olson et al. (2016)

Updike (1990)

Wong et al. (2001)
Mechanically ventilated patients within intensive care.

Mechanically ventilated patients within the intensive care unit.

Mechanically ventilated patients within the intensive care unit.

Adult patients within the intensive care unit. Adult patients undergoing cardiac surgery.

Neurocritical care society members.

Adult patients in ICU

Adult patients in ICU of listening to music of their own choice from the available selection

Anxiety $\uparrow$

60 minutes of music therapy via headphones.

SBP DBP RR $\uparrow$

$\mathrm{HR} \mathrm{SpO}{ }_{2} \rightarrow$

The music group received 30 minute session each day of their choice via headphones

Anxiety $\uparrow$

BP HR RR $\uparrow$

Anxiety $\uparrow$

Serum cortisol $\uparrow$

BP HR RR $\uparrow$

$\mathrm{DBP} \rightarrow$

Music listening

Anxiety $\rightarrow$

Patients listened to music via a music pillow connected to an MP3 player.

MAP HR RR $\mathrm{SpO}_{2} \rightarrow$

Serum cortisol levels $\rightarrow$

Anxiety $\rightarrow$

To understand providers' use of music and TV Practice pattern $\rightarrow$

when caring for patients with an acute neurologic injury whilst in ICU.

Respondents that played music $\uparrow$

Choice of eight programs of classical or contemporary music and listened to 30 minutes via headphones.

30 minutes of music therapy
SBP DBP MAP $\uparrow$

Patients response $\uparrow$

Anxiety $\uparrow$

BP RR 个

Sedation/analgesia $\rightarrow$ 
medRxiv preprint doi: https://doi.org/10.1101/2021.09.11.21263390; this version posted September 13, 2021. The copyright holder for this preprint (which was not certified by peer review) is the author/funder, who has granted medRxiv a license to display the preprint in perpetuity. All rights reserved. No reuse allowed without permission.

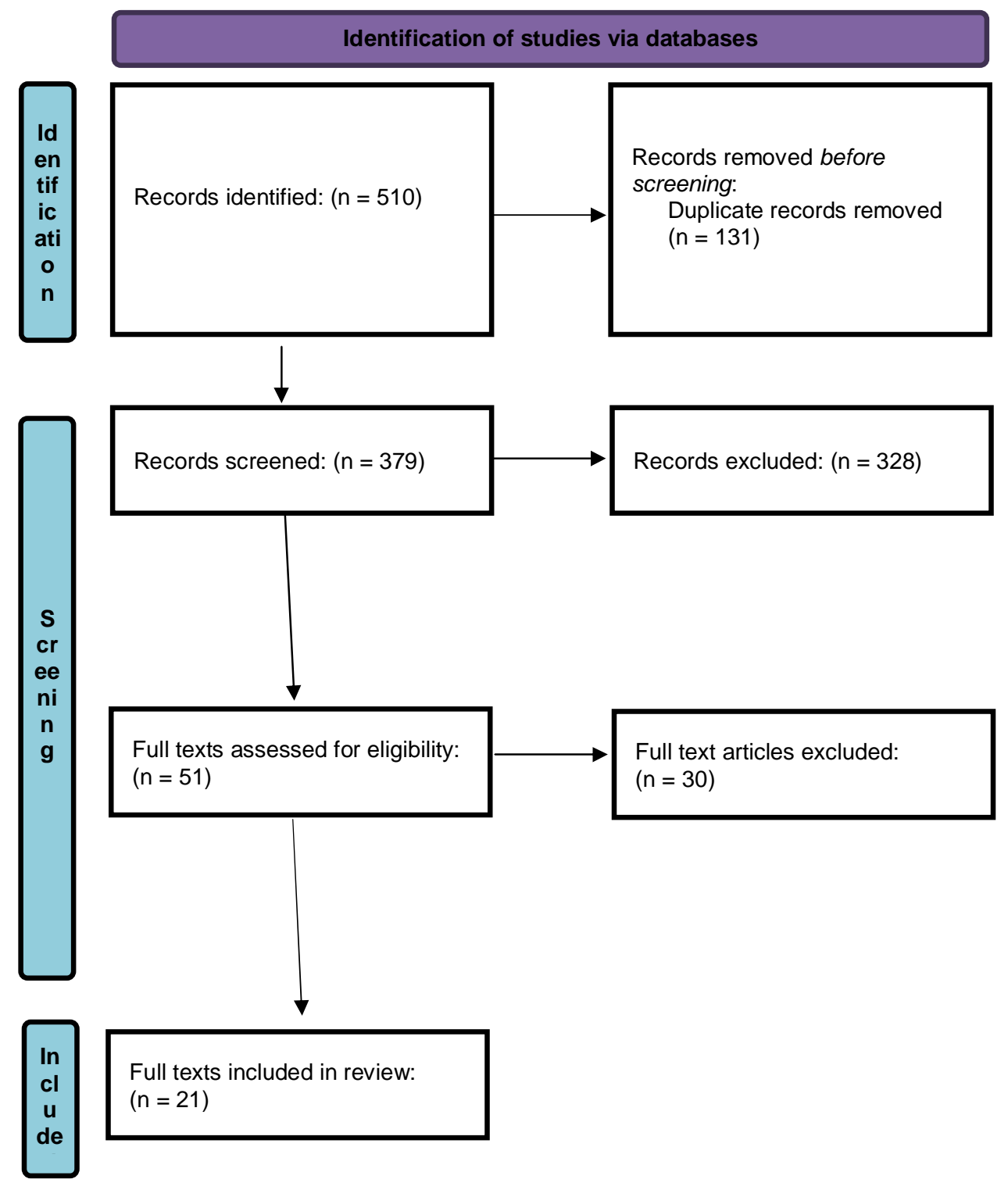

Figure 1 PRIMSA flow diagram 